\title{
Atmospheric Electron Microscope: Limits of Observable Depth
}

\author{
M. Suga ${ }^{*}$, H. Nishiyama ${ }^{*}$, T. Ebihara ${ }^{* *}$, T. Ogura ${ }^{* *}$, C. Sato ${ }^{* *}$ \\ * JEOL Ltd., 1-2 Musashino 3-chome, Akishima, Tokyo 196-8558, Japan \\ ** National Institute of Industrial Science and Technology (AIST), Higashi 1-1-1, Tsukuba, Ibaraki \\ 305-8566, Japan
}

The new Atmospheric Scanning Electron Microscope (ASEM) is able to directly observe cells fixed in a culture medium under atmospheric pressure [1]. In this system, an electron-permeable window made of pressure-resistant film allows an electron beam to be projected from underneath the sample. The electrons backscattered from the samples are captured by a detector positioned below. In the process, since the electron beam is scattered first by the thin film and further by the sample, the depth of observation is restricted. In this study, we theoretically and experimentally investigate the ASEM's depth limits.

An electron trajectory around an ASEM sample is schematically shown in Fig. 1. Electrons are injected into the thin film from beneath the sample and repeatedly scattered in the film and the sample. The electrons are further reflected by a structure made of electron-dense material, and again repeatedly scattered to the surface of the film. Finally, electrons ejected from the surface of the film are captured by the detector. Thus, the maximum observable depth using the ASEM is speculated to be approximately half of the electron penetration depth (range). The range and the electron trajectories are next calculated using Monte Carlo simulation [2] as shown in Fig. 2. For an approximation, a $100 \mathrm{~nm}$ sheet of $\mathrm{Si}_{3} \mathrm{~N}_{4}$ is adopted instead of the $\mathrm{SiN}$ film, while $\mathrm{H}_{2} \mathrm{O}$, the main component of biological specimens, is adopted instead of the sample. The penetration and maximum observable depth are approximately $6 \mathrm{um}$ and $3 \mathrm{um}$ for electrons with an energy of $20 \mathrm{kV}$. Corresponding values for electrons with an energy of $10 \mathrm{kV}$ are $1.5 \mathrm{um}$ and $0.75 \mathrm{um}$.

These calculations are confirmed experimentally. Fig. 3 presents images of COS 7 cells: (a) and (b) show images captured by ASEM, while (c) and (d) show images optically sliced using a laser confocal microscope. In fig. 3(a), the structures indicated by arrows are clearly visible with an electron acceleration voltage of $20 \mathrm{kV}$, although blurred by scattering. In fig. 3(c), the images of the structure are clearly seen in the optically sliced image, indicating that the distance from the structure to the film is approximately $2.83 \mathrm{um}$. The value of $2.83 \mathrm{um}$ is close to the calculated value of the maximum depth (3um) by Monte Carlo simulation. The structure is not seen in Fig. 3(b) with an electron acceleration voltage of $10 \mathrm{kV}$. Here the maximum observation depth is calculated to be $0.75 \mathrm{um}$, which is much smaller than the depth of the structure $(2.83 \mathrm{um})$. Thus, the experimental results are consistent with the calculated results.

\section{References}

[1] H. Nishiyama, M. Suga, M. Koizumi, S. Kitamura, M. Tsuyuki, Y. Ishimori, T. Sato, T. Ogura, C. Sato, New Scanning Electron Microscope Capable of Observing Cells in Solution, to be published in this volume.

[2] P. Hovington, D. Drouin, R. Gauvin, CASINO: a new Monte Carlo code in C Language for electron beam 
interaction - Part I: description of the program, Scanning 19 (1997) 1-14.

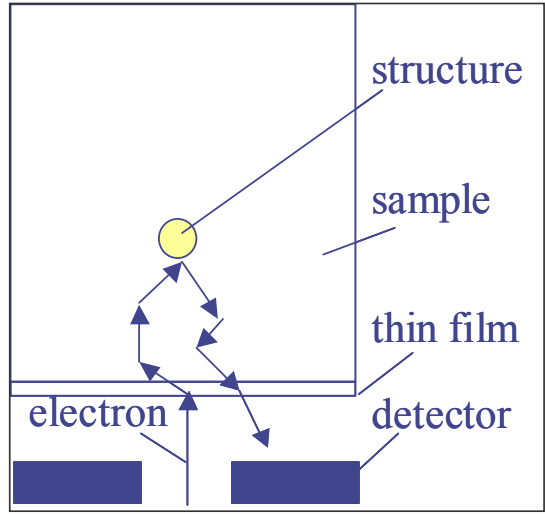

Fig. 1 Electron trajectory around the sample

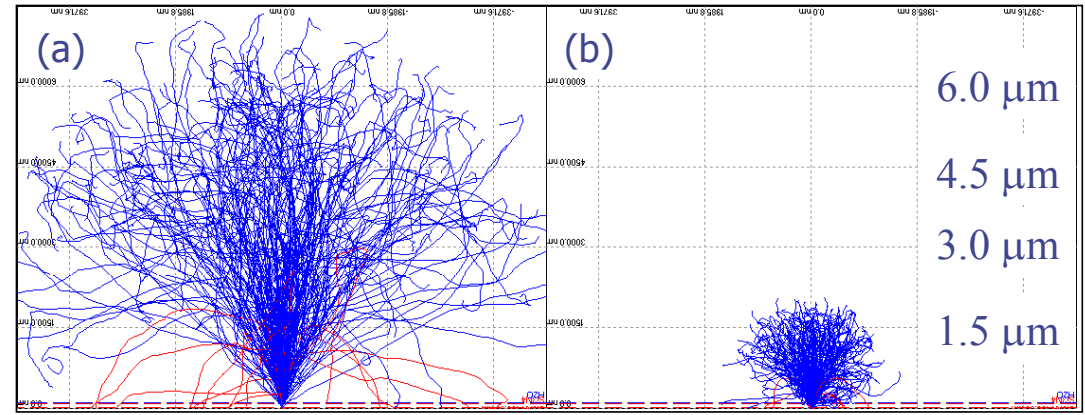

Fig. 2 Electron trajectories calculated using the Monte Carlo method. (a) $20 \mathrm{kV}$ electron acceleration. (b) $10 \mathrm{kV}$ electron acceleration.

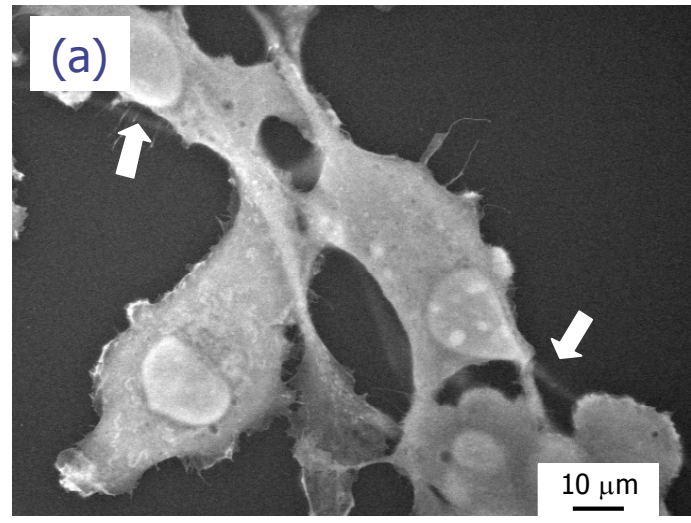

\section{(b)}
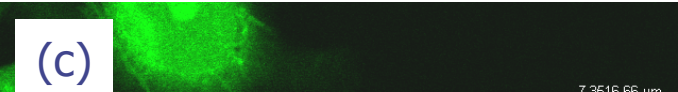

\section{(d)} $10 \mu \mathrm{m}$

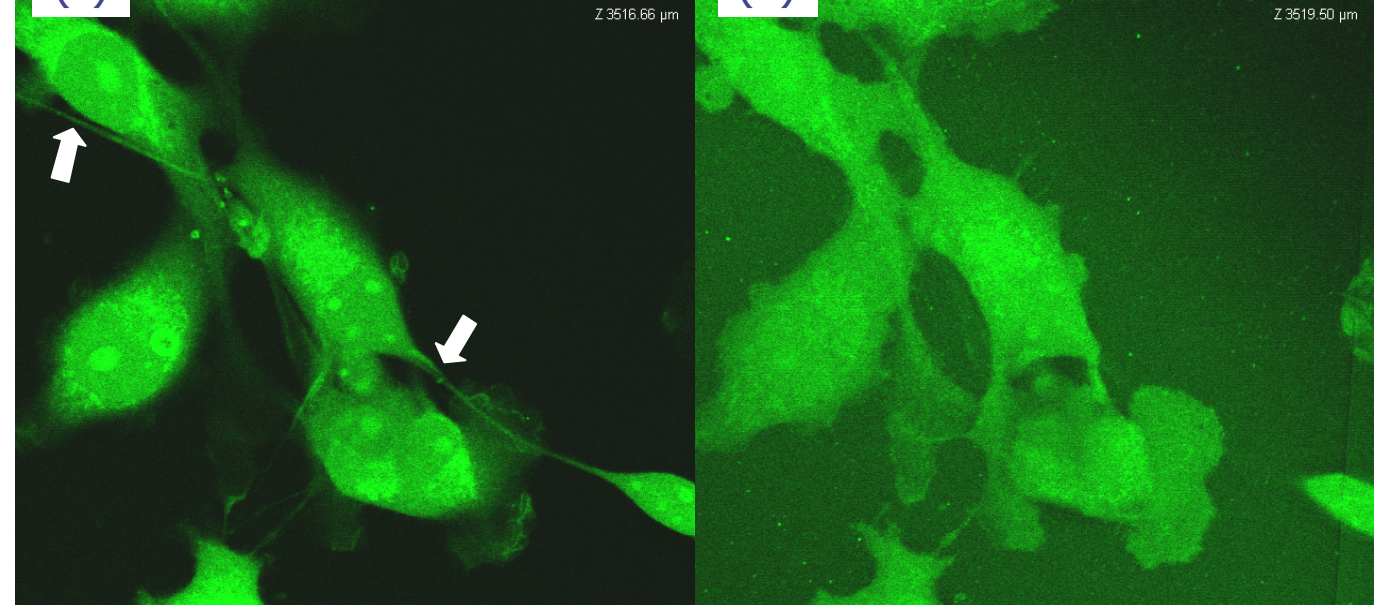

Fig. 3: Images of COS 7 cells; (a), (b) Images captured by ASEM. (a) acceleration voltage of 20 $\mathrm{kV}$, (b) acceleration voltage of $10 \mathrm{kV}$. (c), (d) Images optically sliced using laser confocal microscope. (c): sliced at 2.83 um above the film, (d): sliced at the level of the film. 